\title{
Temperature Dependence of Magnetic Properties of an 18-nm-thick YIG Film Grown by Liquid Phase Epitaxy: Effect of a Pt Overlayer
}

\author{
Nathan Beaulieu*, ${ }^{*}$, Nelly Kervarec ${ }^{\ddagger}$, Nicolas Thiery ${ }^{\S}$, Olivier Klein ${ }^{\S}$, Vladimir Naletov ${ }^{\dagger}, \S, \boldsymbol{\uparrow}$, \\ Hervé Hurdequint $^{\dagger}$, Grégoire de Loubens ${ }^{\dagger}$, Jamal Ben Youssef* and Nicolas Vukadinovic"l \\ *LabSTICC, CNRS, Université de Bretagne Occidentale, 29238 Brest, France \\ ${ }^{\dagger}$ SPEC, CEA, CNRS, Université Paris-Saclay, 91191 Gif-sur-Yvette, France \\ $\ddagger$ Plateforme technologique RMN-RPE, Université de Bretagne Occidentale, 29238 Brest, France \\ §Univ. Grenoble Alpes, CEA, CNRS, Grenoble INP, INAC-Spintec, 38000 Grenoble, France \\ IInstitute of Physics, Kazan Federal University, Kazan 420008, Russian Federation \\ "Dassault Aviation, 92552 Saint-Cloud, France
}

\begin{abstract}
Liquid phase epitaxy of an $18 \mathrm{~nm}$ thick Yttrium Iron garnet (YIG) film is achieved. Its magnetic properties are investigated in the $100-400 \mathrm{~K}$ temperature range, as well as the influence of a $3 \mathrm{~nm}$ thick Pt overlayer on them. The saturation magnetization and the magnetocrystalline cubic anisotropy of the bare YIG film behave similarly to bulk YIG. A damping parameter of only a few $10^{-4}$ is measured, together with a low inhomogeneous contribution to the ferromagnetic resonance linewidth. The magnetic relaxation increases upon decreasing temperature, which can be partly ascribed to impurity relaxation mechanisms. While it does not change its cubic anisotropy, the $\mathbf{P t}$ capping strongly affects the uniaxial perpendicular anisotropy of the YIG film, in particular at low temperatures. The interfacial coupling in the YIG/Pt heterostructure is also revealed by an increase of the linewidth, which substantially grows by lowering the temperature.
\end{abstract}

\section{INTRODUCTION}

Yttrium Iron garnet $\left(\mathrm{Y}_{3} \mathrm{Fe}_{5} \mathrm{O}_{12}, \mathrm{YIG}\right)$ is the marvel material for ferromagnetic resonance (FMR) [1], with the lowest known Gilbert damping parameter, $\alpha=3 \cdot 10^{-5}$ for bulk [2], [3]. Since the 1970s, liquid phase epitaxy (LPE) has been the reference method to grow micrometer-thick YIG films with bulk-like dynamical properties [4], [5], and numerous microwave devices based on the propagation of spin-waves in such films have been developed [6]. In the past years, thin films of YIG have become highly desirable in the context of magnonics [7], [8] and its coupling to spintronics [9] for three main reasons. Firstly, in magnonics, one wishes magnetic films with low damping to ensure large propagation length and with thickness limited to a few tens of nanometers so that fundamental spin-wave modes do not interact with higher order thickness modes, two conditions met in nanometer-thick YIG films [10]. Secondly, coupling YIG magnonics to spintronics was made possible by the discovery that pure spin currents can be transmitted at the interface between YIG (a magnetic insulator) and an adjacent normal metal [11], [12]. In these hybrid bilayers, ultrathin YIG layers are required to enhance the interfacial effects. For instance, the damping of nanometer- thick YIG can be controlled by the spin-orbit torque produced by an electrical current flowing in an adjacent Pt layer [13], leading to the generation of coherent spin-waves above a threshold instability [14]. Thirdly, due to the high resilience of YIG, only nanometer-thick films can be patterned through standard nanofabrication techniques, which is an important asset to design magnonic crystals [15] and engineer the spinwave spectrum of individual YIG nanostructures [16].

Due to this renew of interest for YIG thin films, lots of efforts have been put in the last few years to produce ultrathin films with high epitaxial and dynamical qualities. Pulsed laser deposition (PLD) is a technique of choice to deposit ultrathin YIG layers ablated from stoichiometric targets on $\mathrm{Gd}_{3} \mathrm{Ga}_{5} \mathrm{O}_{12}$ (GGG) substrates [17], [18], [19], [20], [21], [22]. Magnetic properties close to bulk ones [19] and Gilbert damping parameters below $10^{-4}$ have been reported for films thinner than $50 \mathrm{~nm}$ [22], even though such low intrinsic damping often comes at the detriment of the full linewidth due to inhomogeneous broadening [20], [21]. Another method to grow epitaxial nanometer-thick YIG films is off-axis sputtering [23], which can be used to control the strain-induced anisotropy on lattice-mismatched substrates [24]. Finally, even though LPE is not well suited to grow sub-micron thick films, it has been successfully used to produce YIG films with thicknesses in the $100-200 \mathrm{~nm}$ range and damping parameters approaching $10^{-4}$ [25], [26].

Here, we demonstrate that the growth of a YIG film as thin as $18 \mathrm{~nm}$ and of high quality can be achieved by LPE. We present a detailed investigation of its magnetic properties, including relaxation, as a function of temperature, as well as the effect of a $3 \mathrm{~nm}$ thick Pt overlayer on them.

\section{SAMPLE PREPARATION AND PRELIMINARY CHARACTERIZATIONS AT ROOM TEMPERATURE}

The ultrathin YIG film under consideration was grown by LPE from $\mathrm{PbO}$ and $\mathrm{B}_{2} \mathrm{O}_{3}$ flux on a 1 inch (111)-oriented GGG substrate. High-purity oxides $(6 \mathrm{~N})$ were used. The key 

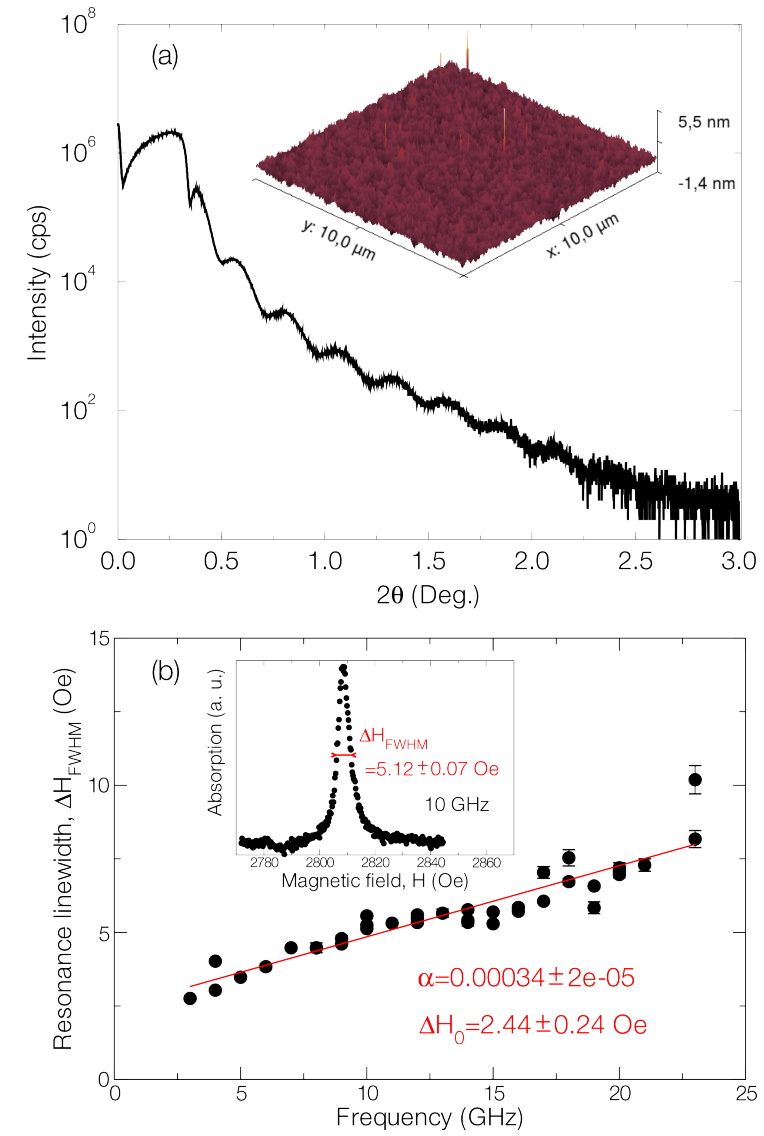

Fig. 1. (a) X-ray reflectometry of the $18 \mathrm{~nm}$ thick LPE grown YIG film. The inset shows the AFM surface topography. (b) Resonance linewidth vs. frequency of the bare YIG layer measured by broadband FMR at room temperature. The inset displays the FMR line at $10 \mathrm{GHz}$.

parameter is the growth rate that depends on the growth temperature. To get an ultrathin YIG film, a very low growth rate is required which means that the growth temperature has to be close to the saturation temperature (small supercooling). For the selected sample, the growth rate was around $1 \mathrm{~nm} / \mathrm{s}$. Another important parameter is the speed of the substrate rotation during the growth process that must be controlled. The quality of the utrathin YIG film was checked by atomic force microscopy (AFM) measurements from which a surface roughness of about $0.25 \mathrm{~nm}$ was determined (see inset in Fig.1(a)). The film thickness of $18 \mathrm{~nm}$ was confirmed by X-ray reflectivity measurements (Fig.1(a)). From the experimental in-plane magnetization curves recorded along the $[11 \overline{0}]$ and $[11 \overline{2}]$ axes (not shown here), several conclusions can be drawn: (i) the saturation magnetization $4 \pi M_{S}$ at room temperature is equal to $1700 \mathrm{G}$, close to the bulk value, (ii) the magnetization curves are isotropic in the film plane, and (iii) the film has a very weak coercive field $\left(H_{c} \simeq 0.3 \mathrm{Oe}\right)$.

Broadband FMR on a millimeter-size slab of the grown film was used to investigate its magnetic relaxation at room temperature. The full width at half maximum (FWHM) of the resonance line measured in in-plane magnetized configuration as a function of the excitation frequency is shown in Fig.1(b).
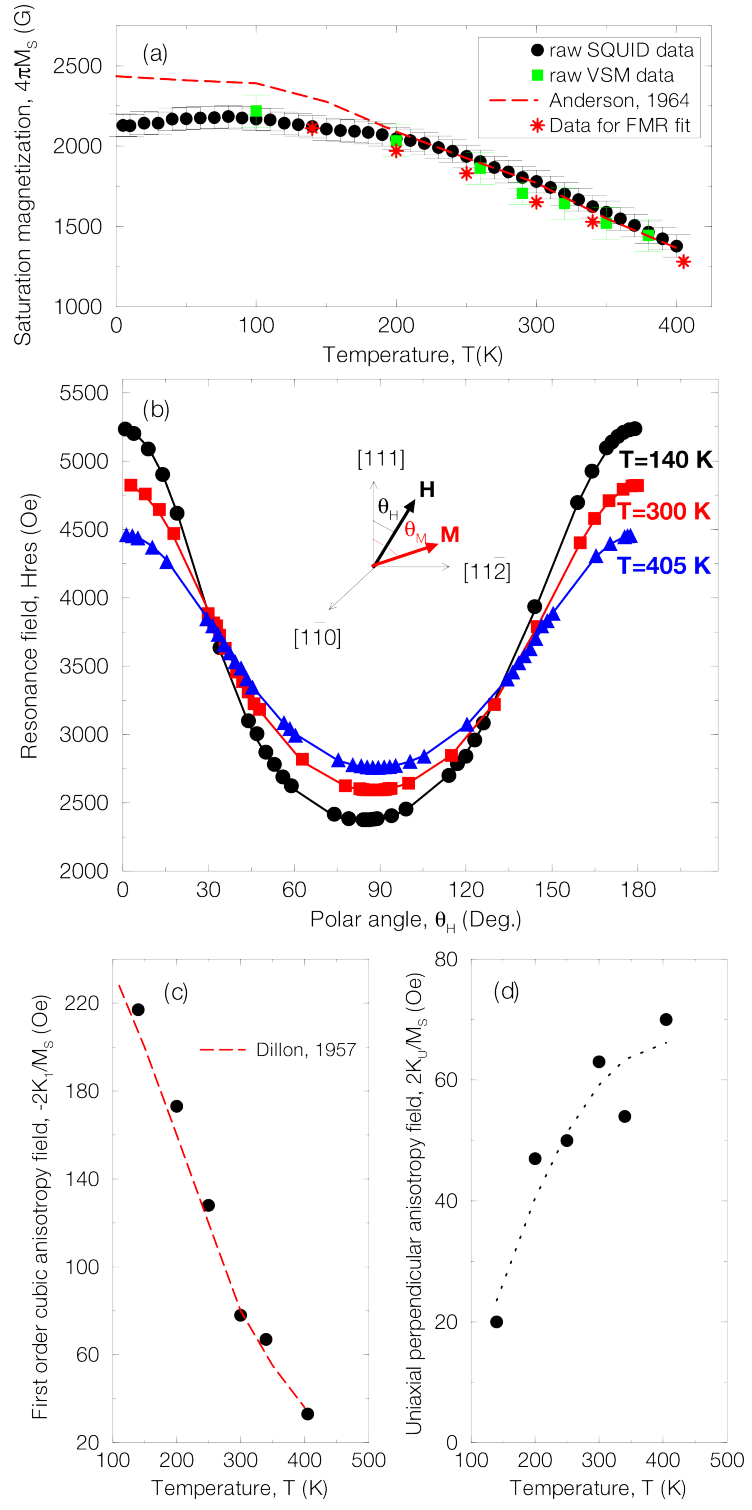

Fig. 2. (a) Dependence of the magnetization of the $18 \mathrm{~nm}$ thick YIG film on temperature. (b) Resonance field vs. polar angle measured at X-band at three different temperatures. (c) Cubic and (d) uniaxial perpendicular anisotropies extracted as a function of temperature. The red dashed lines in (a) and (c) are the dependences for bulk YIG from literature, the dotted line in (d) is a guide to the eye.

The linear dependence of the linewidth on frequency allows to fit a Gilbert damping parameter $\alpha=(3.4 \pm 0.2) \cdot 10^{-4}$ and an inhomogeneous contribution $\Delta H_{0}=2.44 \pm 0.24$ Oe. These values, which are close to those reported on PLD grown YIG films of similar thickness [18], highlight the good dynamical quality of our film.

\section{TEMPERATURE DEPENDENCE OF MAGNETIC PROPERTIES FOR THE BARE YIG FILM}

The temperature dependence of the saturation magnetization for the bare YIG film measured by means of a superconducting quantum interference device (SQUID) magnetometer and a vibrating sample magnetometer (VSM) is displayed in 
Fig.2(a). The $4 \pi M_{S}(T)$ profiles are consistent between the two methods and are in very good agreement with the one reported for a bulk YIG sample [27] for $T \geq 200 \mathrm{~K}$. For lower temperatures, the saturation magnetization for the bulk sample exceeds the one found for our ultrathin YIG film.

Next, the anisotropy constants were extracted from FMR measurements performed in a X-band cavity (magnetic field swept at fixed frequency $f=9.3 \mathrm{GHz}$ ). We note that the slab used in this experiment has a slightly larger inhomogeneous contribution to the linewidth than the one measured by broadband FMR. Using the Makino's procedure [28], the first-order cubic anisotropy constant $K_{1}$, the first-order uniaxial perpendicular anisotropy constant $K_{U}$ and the gyromagnetic ratio $\gamma$ were determined as a function of temperature by exploiting the polar angle variation of the polarizing magnetic field, the $4 \pi M_{S}(T)$ profile being known for that very same slab of film (red stars in Fig.2(a)). Examples of such variations are reported in Fig.2(b) for three temperatures: $T=140 \mathrm{~K}, 300 \mathrm{~K}$, and $405 \mathrm{~K}$, where $\theta_{H}$ is the polar angle of the polarizing magnetic field. $\theta_{H}=0^{\circ}$ corresponds to the film normal ([111] crystallographic axis) and $\theta_{H}=90^{\circ}$ is the in-plane [11̄2] axis. Two remarks can be made. First, the amplitude of the angular variation increases for decreasing temperature. Second, this variation becomes more and more asymmetrical with respect to the film plane as the temperature is lowered. This last point means that the absolute value of $K_{1}$ is enhanced for decreasing temperature. The experimental temperature dependence of $K_{1}$ is displayed in Fig.2(c). The increase of the absolute value of $K_{1}$ for decreasing $T$ satisfactorily matches with the variation found for a bulk YIG sample [29]. On the other hand, the temperature dependence of $K_{U}$ is reported in Fig.2(d). It appears that the sign of $K_{U}$ is positive (easy axis along the film normal) and $K_{U}$ increases with $T$.

The origin of $K_{U}$ in micrometer-thick LPE grown YIG films has been discussed in detail in the eighties. Two main contributions were identified, namely, the growth and the stress anisotropies. For pure YIG films, the growth anisotropy is mainly induced by the $\mathrm{Pb}$ impurities from solvent. It has been established that it raises with the $\mathrm{Pb}$ content [30]. In addition, the $\mathrm{Pb}$ content increases with the supercooling [31]. In our case, the growth of the ultrathin YIG film with a small supercooling prevents a large contribution of the growth anisotropy. On the other hand, the uniaxial stress anisotropy arises from the lattice parameter mismatch between the GGG substrate $\left(a_{s}=12.383 \AA\right)$ and the YIG film $\left(a_{f}=12.376 \AA\right)$. Introducing $\Delta a^{\perp}=\left(a_{s}-a_{f}^{\perp}\right) / a_{s}$ where $a_{f}^{\perp}$ is the lattice parameter of the YIG film in the growth direction, the uniaxial stress anisotropy constant $K_{U}^{s}$ is expressed by: $K_{U}^{s}=(-3 / 2) \lambda_{111} \sigma$ where $\sigma=E /(1-\nu) \Delta a^{\perp}$, where $E$ is the Young's modulus and $\nu$ the Poisson's ratio. For our film grown under tension, $\sigma$ is positive, $\lambda_{111}$ is negative and a positive value of $K_{U}^{s}$ is expected in agreement with the experimental data. An estimate of $K_{U}^{s}$ based on the values of $E, \nu$ and $\lambda_{111}$ for a bulk YIG sample leads to about $40 \%$ of the $K_{U}$ value deduced from FMR measurements. The remaining difference can be ascribed to a surface induced contribution to
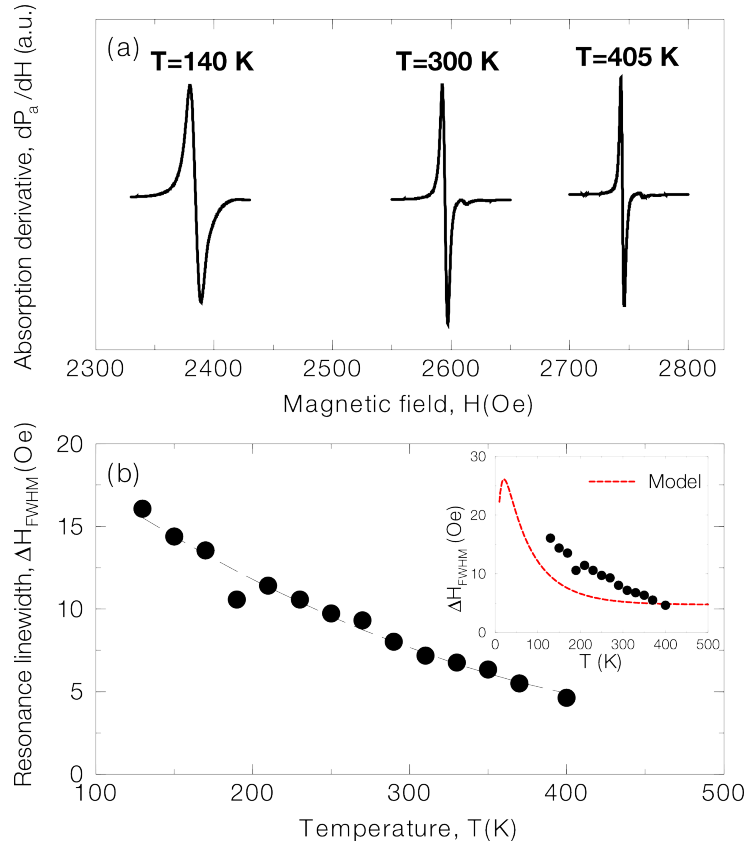

Fig. 3. (a) FMR lines measured at X-band on the bare YIG layer for three different temperatures. (b) FMR linewidth vs. temperature. The inset shows the comparison of the data to the slowly relaxing impurity model (Eq.1).

the uniaxial perpendicular anisotropy, which is also present in ultrathin films. In fact, we have observed at room temperature that $K_{U}$ varies with the YIG thickness, and becomes negative (easy plane) above about $50 \mathrm{~nm}$.

Moreover, a value of the gyromagnetic ratio nearly independent of temperature was extracted from the angular fit of the resonance field, $|\gamma|=1.7685 \pm 0.002 \mathrm{~s}^{-1} \mathrm{Oe}^{-1}$. This value is consistent with the one deduced from the broadband FMR measurements, $|\gamma|=1.771 \pm 0.0007 \mathrm{~s}^{-1} \mathrm{Oe}^{-1}$.

Another interesting feature is the temperature dependence of the magnetic relaxation. Fig.3(a) shows the absorption spectra (derivative form) versus magnetic field recorded at $9.3 \mathrm{GHz}$ in the parallel configuration for three temperatures. These spectra reveal that the FMR linewidth increases for decreasing temperature. The value of $\Delta H_{\text {FWHM }}$ at $140 \mathrm{~K}$ is nearly four times greater than the one at $405 \mathrm{~K}$. Such a behavior has been recently reported on ultrathin YIG films grown either by a spin coating method [32] or by off-axis sputtering [33], and on YIG spheres [34]. The broader temperature range probed in these studies allows to evidence a peak-like maximum in the temperature dependence of the FMR linewidth. The position of this peak is frequency dependent and is located around $T=25 \mathrm{~K}$ at $\mathrm{X}$-band. Based on these observations, the origin of the resonant temperature dependent linewidth was ascribed to the slowly relaxing impurity mechanism extensively investigated on bulk YIG samples in the sixties [2], [3]. The existence of rare earth or $\mathrm{Fe}^{2+}$ impurities induced during the growth process was put forward. In both cases, the contribution of the slowly relaxing impurity mechanism to the 


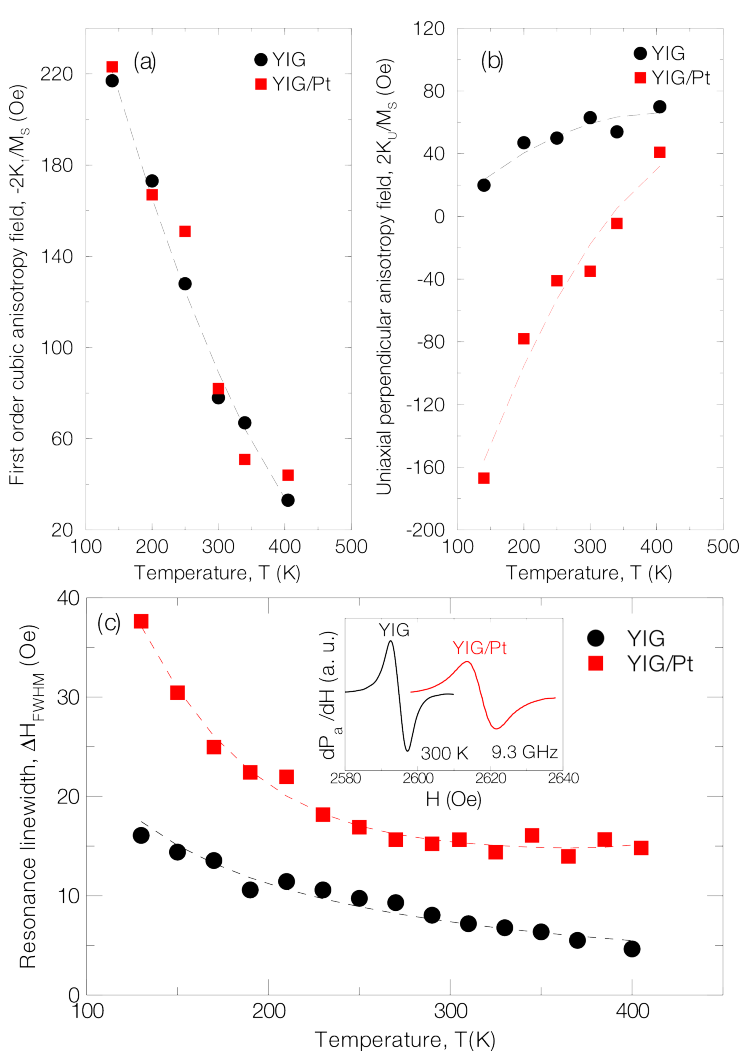

Fig. 4. Dependences on temperature of (a) cubic anisotropy, (b) uniaxial perpendicular anisotropy and (c) resonance linewidth for both the bare YIG layer (black dots) and the YIG layer capped with $3 \mathrm{~nm}$ Pt (red squares). The inset shows the FMR lines measured at $300 \mathrm{~K}$ on both samples. All dashed lines are guides to the eye.

linewidth can be described by the following expression [33]:

$$
\Delta H_{S R}=A(T) \frac{\omega \tau}{1+(\omega \tau)^{2}},
$$

where $A(T)$ is a frequency-independent prefactor and $\tau$ a temperature-dependent time constant. The total FMR linewidth $\Delta H_{\mathrm{FWHM}}=\Delta H_{0}+\frac{4 \pi \alpha f}{|\gamma|}+\Delta H_{S R}$ is displayed in the inset in Fig.3(b) (red dashed curve). It can be seen that the experimental temperature dependence of the FMR linewidth cannot be fully reproduced by the model, where only $\Delta H_{S R}$ is a function of temperature. Other relaxation mechanisms seem to contribute to the FMR linewidth. As highlighted in Ref.[26], the existence of a transition layer between LPE grown YIG films and the GGG substrate, whose thickness is estimated to be around $5 \mathrm{~nm}$ (nearly a third of our film thickness), could produce additional relaxation channels. For our ultrathin film, a sizeable surface induced contribution to the linewidth can also be expected.

\section{TEMPERATURE DEPENDENCE OF MAGNeTIC PROPERTIES FOR THE YIG/PT HETEROSTRUCTURE}

Next, we are interested in the influence of a thin overlayer of $\mathrm{Pt}$ on the magnetic properties of our ultrathin YIG film. Using electron beam evaporation, a $3 \mathrm{~nm}$ thick Pt layer was evaporated on top of the YIG film after a very soft in situ dry etching of its surface. Similarly to the bare YIG film, the obtained YIG/Pt heterostructure was then studied at Xband as a function of temperature. The main results of this characterization are compared to those obtained on the bare YIG film in Fig.4. In order to extract the anisotropy constants of the YIG/Pt bilayer, we used the same $4 \pi M_{S}(T)$ profile as in Fig.2(a), since it is known that no magnetic moment is induced in Pt by proximity effects at ferrites/Pt interfaces [35]. As can be seen in Fig.4(a), the Pt overlayer does not change the cubic anisotropy of the bare YIG film, which is expected from its bulk magnetocrystalline origin. In contrast, it does strongly affect the uniaxial perpendicular anisotropy, which becomes negative (easy plane) at low temperature, as shown in Fig.4(b). It means that an interfacial mechanism [36] of spin-orbit origin changes the surface anisotropy component of $K_{U}$ discussed above for the bare YIG film. The gyromagnetic ratio remains unaffected by the Pt overlayer within our determination uncertainty.

As expected, the interfacial coupling between YIG and Pt also leads to the increase of the FMR linewidth [37], [38], which is obvious from the comparison presented in Fig.4(c). At $300 \mathrm{~K}$, the measured linewidth is nearly doubled by the deposition of $\mathrm{Pt}$ on top of YIG. To prove its interfacial origin, we have checked that this enhancement is inversely proportional on the YIG thickness by varying the latter in the $15-200 \mathrm{~nm}$ range (not shown here). It is interesting to note that the increase of the linewidth due to the $\mathrm{Pt}$ overlayer is not constant as a function of temperature. The increment in the damping depends both on the strength of the interfacial coupling (whose microscopic origin is rarely discussed, most often, it is parametrized by the so-called spin mixing conductance of the hybrid interface) and on the spin transport parameters in the Pt layer (the thickness of the latter being here comparable to the spin diffusion length in $\mathrm{Pt}$ at room temperature [39]). These two contributions can be temperature dependent, leading to a non trivial behavior of the increment in the damping versus temperature. As a matter of fact, the latter seems to be minimum around $250 \mathrm{~K}$, and its rapid increase below this temperature should be confirmed by measurements at even lower temperature [32].

\section{CONClusion}

In sum, this study demonstrates that the LPE growth method can be used to produce YIG films with thickness below $20 \mathrm{~nm}$ and good structural parameters. Surface induced contributions to the anisotropy and relaxation are present in these ultrathin films. The effect of a Pt overlayer proves that the interfacial coupling with this heavy metal is efficient. In fact, similar batches of ultrathin LPE grown YIG films have already been used to study the transport of angular momentum in non-local YIG/Pt devices using spin-to-charge interconversion [40], [41].

\section{ACKNOWLEDGMENT}

We would like to thank Philippe Eliès and François Michaux from the PIMM-DRX platform at UBO for their help with the AFM characterization and the X-ray reflectometry. 


\section{REFERENCES}

[1] V. Cherepanov, I. Kolokolov, and V. L'vov, "The saga of YIG: spectra, thermodynamics, interaction and relaxation of magnons in a complex magnet," Physics Reports, vol. 229, p. 81, 1993.

[2] E. G. Spencer, R. C. LeCraw, and A. M. Clogston, "Low-temperature line-width maximum in yttrium iron garnet," Phys. Rev. Lett., vol. 3, pp 32-33, 1959.

[3] M. Sparks, Ferromagnetic relaxation theory. New York: McGraw-Hill, 1964.

[4] R. D. Henry, P. J. Besser, D. M. Heinz, and J. E. Mee, "Ferromagnetic resonance properties of LPE YIG films," IEEE Trans. Magn., vol. 9, pp. 535-537, 1973.

[5] H. Wei and W. Wang, "The growth of LPE YIG films with narrow fmr linewidth,” IEEE Trans. Magn., vol. 20, no. 5, pp. 1222-1223, 1984.

[6] J.-P. Castera, "State of the art in design and technology of MSW devices (invited)," J. Appl. Phys., vol. 55, pp. 2506-2511, 1984.

[7] V. V. Kruglyak, S. O. Demokritov, and D. Grundler, "Magnonics," J. Phys. D, vol. 43, p. 264001, 2010.

[8] A. A. Serga, A. V. Chumak, and B. Hillebrands, "YIG magnonics," Journal of Physics D: Applied Physics, vol. 43, p. 264002, 2010.

[9] A. V. Chumak, V. I. Vasyuchka, A. A. Serga, and B. Hillebrands, "Magnon spintronics," Nature Phys., vol. 11, pp. 453-461, 2015.

[10] H. Yu, O. d'Allivy Kelly, V. Cros, R. Bernard, P. Bortolotti, A. Anane, F. Brandl, R. Huber, I. Stasinopoulos, and D. Grundler, "Magnetic thin-film insulator with ultra-low spin wave damping for coherent nanomagnonics," Sci. Rep., vol. 4, p. 6848, 2014.

[11] Y. Kajiwara, K. Harii, S. Takahashi, J. Ohe, K. Uchida, M. Mizuguchi, H. Umezawa, H. Kawai, K. Ando, K. Takanashi, S. Maekawa, and E. Saitoh, "Transmission of electrical signals by spin-wave interconversion in a magnetic insulator," Nature, vol. 464, pp. 262-266, 2010.

[12] L. J. Cornelissen, J. Liu, R. A. Duine, J. Ben Youssef, and B. J. van Wees, "Long-distance transport of magnon spin information in a magnetic insulator at room temperature," Nature Phys., vol. 11, pp. 1022-1026, 2015.

[13] A. Hamadeh, O. d'Allivy Kelly, C. Hahn, H. Meley, R. Bernard, A. H. Molpeceres, V. V. Naletov, M. Viret, A. Anane, V. Cros, S. O. Demokritov, J. L. Prieto, M. Muñoz, G. de Loubens, and O. Klein, "Full control of the spin-wave damping in a magnetic insulator using spin-orbit torque," Phys. Rev. Lett., vol. 113, p. 197203, 2014.

[14] M. Collet, X. de Milly, O. d'Allivy Kelly, V. Naletov, R. Bernard, P. Bortolotti, J. Ben Youssef, V. Demidov, S. Demokritov, J. Prieto, M. Muñoz, V. Cros, A. Anane, G. de Loubens, and O. Klein, "Generation of coherent spin-wave modes in yttrium iron garnet microdiscs by spinorbit torque," Nature Commun., vol. 7, p. 10377, 2016.

[15] M. Krawczyk and D. Grundler, "Review and prospects of magnonic crystalsand devices with reprogrammable band structure," J. Phys.: Condens. Matter, vol. 26, no. 12, p. 123202, 2014.

[16] C. Hahn, V. V. Naletov, G. de Loubens, O. Klein, O. d'Allivy Kelly, A. Anane, R. Bernard, E. Jacquet, P. Bortolotti, V. Cros, J. L. Prieto, and M. Muñoz, "Measurement of the intrinsic damping constant in individual nanodisks of $\mathrm{Y}_{3} \mathrm{Fe}_{5} \mathrm{O}_{12}$ and $\mathrm{Y}_{3} \mathrm{Fe}_{5} \mathrm{O}_{12} \mid \mathrm{Pt}$," Appl. Phys. Lett., vol. 104, p. $152410,2014$.

[17] Y. Sun, Y.-Y. Song, H. Chang, M. Kabatek, M. Jantz, W. Schneider, M. Wu, H. Schultheiss, and A. Hoffmann, "Growth and ferromagnetic resonance properties of nanometer-thick yttrium iron garnet films," Appl. Phys. Lett., vol. 101, p. 152405, 2012.

[18] O. d'Allivy Kelly, A. Anane, R. Bernard, J. Ben Youssef, C. Hahn, A. H. Molpeceres, C. Carretero, E. Jacquet, C. Deranlot, P. Bortolotti, R. Lebourgeois, J.-C. Mage, G. de Loubens, O. Klein, V. Cros, and A. Fert, "Inverse spin hall effect in nanometer-thick yttrium iron garnet/Pt system," Appl. Phys. Lett., vol. 103, p. 082408, 2013.

[19] M. C. Onbasli, A. Kehlberger, D. H. Kim, G. Jakob, M. Klui, A. V. Chumak, B. Hillebrands, and C. A. Ross, "Pulsed laser deposition of epitaxial yttrium iron garnet films with low Gilbert damping and bulklike magnetization," APL Mater, vol. 2, p. 106102, 2014.

[20] H. Chang, P. Li, W. Zhang, T. Liu, A. Hoffmann, L. Deng, and M. Wu, "Nanometer-thick yttrium iron garnet films with extremely low damping," IEEE Magn. Lett., vol. 5, p. 6700104, 2014.

[21] L. V. Lutsev, A. M. Korovin, V. E. Bursian, S. V. Gastev, V. V. Fedorov, S. M. Suturin, and N. S. Sokolov, "Low-relaxation spin waves in lasermolecular-beam epitaxy grown nanosized yttrium iron garnet films," Appl. Phys. Lett., vol. 108, no. 18, 2016.
[22] C. Hauser, T. Richter, N. Homonnay, C. Eisenschmidt, M. Qaid, H. Deniz, D. Hesse, M. Sawicki, S. G. Ebbinghaus, and G. Schmidt, "Yttrium iron garnet thin films with very low damping obtained by recrystallization of amorphous material," Sci. Rep., vol. 6, p. 20827, 2016.

[23] H. L. Wang, C. H. Du, Y. Pu, R. Adur, P. C. Hammel, and F. Y. Yang, "Large spin pumping from epitaxial $\mathrm{Y}_{3} \mathrm{Fe}_{5} \mathrm{O}_{12}$ thin films to $\mathrm{Pt}$ and $\mathrm{W}$ layers," Phys. Rev. B, vol. 88, p. 100406, 2013.

[24] H. Wang, C. Du, P. C. Hammel, and F. Yang, "Strain-tunable magnetocrystalline anisotropy in epitaxial $\mathrm{Y}_{3} \mathrm{Fe}_{5} \mathrm{O}_{12}$ thin films," Phys. Rev. $B$, vol. 89, p. $134404,2014$.

[25] C. Hahn, G. de Loubens, O. Klein, M. Viret, V. V. Naletov, and J. Ben Youssef, "Comparative measurements of inverse spin hall effects and magnetoresistance in YIG/Pt and YIG/Ta," Phys. Rev. B, vol. 87, p. 174417, 2013.

[26] C. Dubs, O. Surzhenko, R. Linke, A. Danilewsky, U. Brckner, and J. Dellith, "Sub-micrometer yttrium iron garnet LPE films with low ferromagnetic resonance losses," J. Phys. D: Appl. Phys., vol. 50, no. 20, p. 204005, 2017.

[27] E. E. Anderson, "Molecular field model and the magnetization of YIG," Phys. Rev., vol. 134, pp. A1581-A1585, 1964.

[28] H. Makino and Y. Hidaka, "Determination of magnetic anisotropy constants for bubble garnet epitaxial films using field orientation dependence in ferromagnetic resonances," Mat. Res. Bull., vol. 16, pp. 957-966, 1981.

[29] J. F. Dillon, "Ferrimagnetic resonance in yttrium iron garnet," Phys. Rev., vol. 105, pp. 759-760, 1957.

[30] J. Desvignes, D. Mahasoro, and H. Le Gall, "Narrow fmr linewidth dependence on growth conditions in LPE YIG films," IEEE Trans. Magn., vol. 23, no. 5, pp. 3724-3726, 1987.

[31] P. Hansen, K. Witter, and W. Tolksdorf, "Magnetic and magneto-optic properties of lead- and bismuth-substituted yttrium iron garnet films," Phys. Rev. B, vol. 27, pp. 6608-6625, 1983.

[32] E. Shigematsu, Y. Ando, R. Ohshima, S. Dushenko, Y. Higuchi, T. Shinjo, H. J. von Bardeleben, and M. Shiraishi, "Significant reduction in spin pumping efficiency in a platinum/yttrium iron garnet bilayer at low temperature," Appl. Phys. Express, vol. 9, no. 5, p. 053002, 2016.

[33] C. L. Jermain, S. V. Aradhya, N. D. Reynolds, R. A. Buhrman, J. T. Brangham, M. R. Page, P. C. Hammel, F. Y. Yang, and D. C. Ralph, "Increased low-temperature damping in yttrium iron garnet thin films," Phys. Rev. B, vol. 95, p. 174411, 2017.

[34] H. Maier-Flaig, S. Klingler, C. Dubs, O. Surzhenko, R. Gross, M. Weiler, H. Huebl, and S. T. B. Goennenwein, "Temperature-dependent magnetic damping of yttrium iron garnet spheres," Phys. Rev. B, vol. 95, p. 214423, 2017

[35] M. Collet, R. Mattana, J.-B. Moussy, K. Ollefs, S. Collin, C. Deranlot, A. Anane, V. Cros, F. Petroff, F. Wilhelm, and A. Rogalev, "Investigating magnetic proximity effects at ferrite/Pt interfaces," Appl. Phys. Lett., vol. 111, no. 20, p. 202401, 2017.

[36] C. Tang, Q. Song, C.-Z. Chang, Y. Xu, Y. Ohnuma, M. Matsuo, Y. Liu, W. Yuan, Y. Yao, J. S. Moodera, S. Maekawa, W. Han, and J. Shi, "Dirac surface state-modulated spin dynamics in a ferrimagnetic insulator at room temperature," Sci. Adv., vol. 4, no. 6, 2018.

[37] H. Hurdequint, "FMR studies of single permalloy layers sandwiched by Au,” J. Magn. Magn. Mater, vol. 310, pp. 2061-2063, 2007.

[38] B. Heinrich, C. Burrowes, E. Montoya, B. Kardasz, E. Girt, Y.-Y. Song, Y. Sun, and M. Wu, "Spin pumping at the magnetic insulator (YIG)/normal metal (Au) interfaces," Phys. Rev. Lett., vol. 107, no. 6, p. 066604, 2011.

[39] V. Castel, N. Vlietstra, J. Ben Youssef, and B. J. van Wees, "Platinum thickness dependence of the inverse spin-hall voltage from spin pumping in a hybrid yttrium iron garnet/platinum system," Appl. Phys. Lett., vol. 101, p. 132414, 2012.

[40] N. Thiery, A. Draveny, V. V. Naletov, L. Vila, J. P. Attané, C. Beigné, G. de Loubens, M. Viret, N. Beaulieu, J. Ben Youssef, V. E. Demidov, S. O. Demokritov, A. N. Slavin, V. S. Tiberkevich, A. Anane, P. Bortolotti, V. Cros, and O. Klein, "Nonlinear spin conductance of yttrium iron garnet thin films driven by large spin-orbit torque," Phys. Rev. B, vol. 97, p. 060409, 2018.

[41] N. Thiery, V. V. Naletov, L. Vila, A. Marty, A. Brenac, J.-F. Jacquot, G. de Loubens, M. Viret, A. Anane, V. Cros, J. Ben Youssef, N. Beaulieu, V. E. Demidov, B. Divinskiy, S. O. Demokritov, and O. Klein, "Electrical properties of epitaxial yttrium iron garnet ultrathin films at high temperatures," Phys. Rev. B, vol. 97, p. 064422, 2018. 